\title{
Statistical Analysis of Radio Propagation Channel in Ruins Environment
}

\author{
Jiao He, Er-Ping Li, Sai-Qiong Zhou, and Kun Liao \\ Department of Information Science and Electronic Engineering, Zhejiang University, Hangzhou 310027, China
}

Correspondence should be addressed to Er-Ping Li; liep@zju.edu.cn

Received 10 January 2015; Revised 17 March 2015; Accepted 17 March 2015

Academic Editor: Christoph F. Mecklenbräuker

Copyright (C) 2015 Jiao He et al. This is an open access article distributed under the Creative Commons Attribution License, which permits unrestricted use, distribution, and reproduction in any medium, provided the original work is properly cited.

\begin{abstract}
The cellphone based localization system for search and rescue in complex high density ruins has attracted a great interest in recent years, where the radio channel characteristics are critical for design and development of such a system. This paper presents a spatial smoothing estimation via rotational invariance technique (SS-ESPRIT) for radio channel characterization of high density ruins. The radio propagations at three typical mobile communication bands $(0.9,1.8$, and $2 \mathrm{GHz})$ are investigated in two different scenarios. Channel parameters, such as arrival time, delays, and complex amplitudes, are statistically analyzed. Furthermore, a channel simulator is built based on these statistics. By comparison analysis of average excess delay and delay spread, the validation results show a good agreement between the measurements and channel modeling results.
\end{abstract}

\section{Introduction}

As mobile phone is becoming more and more widely used nowadays, a phone-based localization system for search and rescue in emergency situations, such as earthquakes, avalanches, and landslides, has received considerable attention recently [1-4]. To develop and implement such a system, a comprehensive study on the radio propagation behaviors in disaster scenarios is required, especially for system configuration and localization. However, there is a lack of comprehensive study on this issue so far. For pedestrian localization of victims in ruins, the radio propagation characteristics between the cellphone and handheld detection device were studied in [5]. In [6], the base station (BS) to mobile station (MS) radio propagation channel was investigated in two simple artificial ruins, where the rubbles covered only a small area around transmitter in an open field. In actual disaster scenarios, large area of ruins is very common, and the disaster channel suffers extremely complicated fading. To evaluate the influence of large amounts of scatterers and to ensure effective implementation of the phone-based localization system, further researches are required to characterize the radio propagation behaviors in ruins, and investigation of channel modeling method for such complex scenarios is very critical.

For characterization of the radio propagation channel, wideband measurement can be conducted to derive the impulse responses by parameter estimation. Traditionally, the inverse Fourier transform method is utilized for this application. However, the order of Fourier modeling is too large and less efficient for simulation $[6,7]$. Over the past years, conventional spectral estimation methods [8] have been applied for channel modeling. In [7], a low-order autoregressive model was employed to model the indoor radio propagation channel. In [9], three parameter estimation methods, including minimum norm [10], autoregressive model, and frequency response matching method, were compared. In the dense ruins environment, a lot of interferences show up due to poor channel conditions. Under this circumstance, the aforementioned parameter estimation methods are no longer effective. In this study, the ESPRIT method is applied for modeling of the radio propagation channel in dense ruins. As a high efficiency spectral estimation approach, the ESPRIT method has been utilized for resolution enhancement in several specific situations, such as in $[11,12]$, where it was applied to estimate the time delays for ground penetrating radar.

In this paper, the SS-ESPRIT approach is utilized to derive the parameters of channel impulse responses in ruins. Through wideband measurements, the parameters are statistically analyzed in two typical ruins scenarios at three communication bands: $0.9,1.8$, and $2 \mathrm{GHz}$. Based on the statistics, a channel simulator is built and the statistical properties of two key channel parameters, including average excess delay and delay spread, are analyzed. 
TABLE 1: Comparison of the NRMSEs of different methods at different SNRs.

\begin{tabular}{|c|c|c|c|c|c|c|}
\hline \multirow[b]{2}{*}{ SNR } & \multicolumn{3}{|c|}{ NRMSE } & \multicolumn{3}{|c|}{ Model order } \\
\hline & $\begin{array}{c}\text { Burg- } \\
\text { autoregressive }\end{array}$ & $\begin{array}{c}\text { Minimum } \\
\text { norm }\end{array}$ & SS-ESPRIT & $\begin{array}{c}\text { Burg- } \\
\text { autoregressive }\end{array}$ & $\begin{array}{c}\text { Minimum } \\
\text { norm }\end{array}$ & SS-ESPRIT \\
\hline High & 0.003 & 0.000 & 0.000 & 129 & 177 & 33 \\
\hline $20 \mathrm{~dB}$ & 0.674 & 0.586 & 0.140 & 134 & 178 & 48 \\
\hline $10 \mathrm{~dB}$ & 1.772 & 0.992 & 0.397 & 128 & 165 & 48 \\
\hline
\end{tabular}

\section{Analysis Methodology and Validation}

2.1. Multipath Propagation Model. The multipath propagation channel impulse response model in frequency domain is expressed as follows:

$$
H(f)=\sum_{k=1}^{K} a_{k} e^{j \theta_{k}} e^{-j 2 \pi f \tau_{k}}+n(f)
$$

where $K, a_{k}, \theta_{k}$, and $\tau_{k}$ are the number, amplitudes, phases, and delays of multipath components, respectively, and $n(f)$ denotes additive white Gaussian noise.

2.2. ESPRIT-Based Channel Parameter Estimation. Through measurements, we can obtain the discrete channel frequency responses $H\left(f_{n}\right)$ with $n=1,2, \ldots, N$ and $f_{n}=f_{0}+n \cdot \Delta f$, where $f_{0}$ is the start frequency and $\Delta f$ is the sampling interval. Afterwards, parameter estimation method is applied to determine the four parameters in (1): $K, a_{k}, \theta_{k}$, and $\tau_{k}$.

The measured channel frequency response is denoted as follows:

$$
\mathbf{H}=\mathbf{A c}+\mathbf{n},
$$

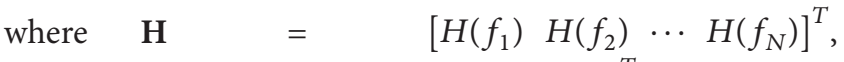

$$
\begin{aligned}
& \text { c }=\left[\begin{array}{lllll}
a_{1} e^{j \theta_{1}} & a_{2} e^{j \theta_{2}} & \cdots & a_{K} e^{j \theta_{K}}
\end{array}\right]^{T}, \quad \mathbf{n}= \\
& {\left[\begin{array}{llll}
n\left(f_{1}\right) & n\left(f_{2}\right) & \cdots & n\left(f_{N}\right)
\end{array}\right]^{T} \text {, and }} \\
& \mathbf{A}=\left[\begin{array}{cccc}
e^{-j 2 \pi f_{1} \tau_{1}} & e^{-j 2 \pi f_{1} \tau_{2}} & \cdots & e^{-j 2 \pi f_{1} \tau_{K}} \\
e^{-j 2 \pi f_{2} \tau_{1}} & e^{-j 2 \pi f_{2} \tau_{2}} & \cdots & e^{-j 2 \pi f_{2} \tau_{K}} \\
\vdots & \vdots & \ddots & \vdots \\
e^{-j 2 \pi f_{N} \tau_{1}} & e^{-j 2 \pi f_{N} \tau_{2}} & \cdots & e^{-j 2 \pi f_{N} \tau_{K}}
\end{array}\right] .
\end{aligned}
$$

Let $\mathbf{H}_{\mathbf{1}}=\left[\begin{array}{llll}H\left(f_{1}\right) & H\left(f_{2}\right) & \cdots & H\left(f_{N-1}\right)\end{array}\right]^{T}, \mathbf{H}_{\mathbf{1}}=\mathbf{A}_{\mathbf{1}} \mathbf{c}+\mathbf{n}_{\mathbf{1}}$, and $\mathbf{H}_{\mathbf{2}}=\left[\begin{array}{lllll}H\left(f_{2}\right) & H\left(f_{3}\right) & \cdots & H\left(f_{N}\right)\end{array}\right]^{T}$; then we can derive

$$
H_{2}=A_{2} c+n_{2}=A_{1} \Phi c+n_{2},
$$

where

$$
\Phi=\operatorname{diag}\left(e^{-j 2 \pi \Delta f \tau_{1}}, e^{-j 2 \pi \Delta f \tau_{2}}, \ldots, e^{-j 2 \pi \Delta f \tau_{K}}\right) .
$$

The ESPRIT estimation is a nonparametric method, which is based on eigenvalue decomposition of a specific correlation matrix, and the largest $K$ eigenvalues correspond to signal subspace, whereas the remaining eigenvalues correspond to noise subspace. The $\tau_{k}$ in $\Phi$ can be derived directly by three ESPRIT-based approaches: total least squares [13], time-shift [14], and spatial smoothing. In this study, the SSESPRIT method shows a superior performance compared to the other two, which will be illustrated in Section 4. For channel modeling, the number of multipath components, $K$, should be determined first and the minimum description length method in [15] is recommended for estimation, which is given as follows:

$$
K=\min _{M} N(L-M) \log \left(\frac{f_{1}(M)}{f_{2}(M)}\right)+f_{3}(M, N),
$$

where $N$ is the independent samples, $L$ is the total number of eigenvalues of the correlation matrix, $f_{1}(M)$ and $f_{2}(M)$ are the arithmetic and geometric averages of the smallest $L-$ $M$ eigenvalues, respectively, and $f_{3}(M, N)=0.5 M(2 N-$ M) $\log (N)$.

After estimating $K$ and $\tau_{k}$ in (1), the values of $a_{k}$ and $\theta_{k}$ are determined by solving the least squares solution of the equation $\mathbf{H}=\mathbf{A c}$, which is referred to as extended Prony's method [9].

2.3. Model Validation by Simulation. To validate the SSESPRIT method, a simulation is performed, and it is compared with the other two parameter estimation methods: Burg-autoregressive method and minimum norm. The normalized root mean square error (NRMSE) is utilized to evaluate the performance of these methods, which is calculated by the following expression:

$$
\mathrm{NRMSE}=\sqrt{\frac{\sum_{n=1}^{N}\left|\widehat{H}\left(f_{n}\right)-H\left(f_{n}\right)\right|^{2}}{\sum_{n=1}^{N}\left|H\left(f_{n}\right)\right|^{2}}},
$$

where $\widehat{H}\left(f_{n}\right)$ is the reconstructed responses based on estimated parameters and $H\left(f_{n}\right)$ is the measured responses.

The simulated channel frequency responses are at the $900 \mathrm{MHz}$ frequency band with a bandwidth of $400 \mathrm{MHz}$. Moreover, the number of multipath components is set to be 5 and the delays and amplitudes are uniformly distributed in the range between [100 ns, $150 \mathrm{~ns}]$ and $[0,0.6]$, respectively. Based on these assumptions, the "measured" channel frequency responses are constructed according to (1), which is referred to as "original data" in the following description. By 200 times of simulation, the average NRMSEs and model orders at different signal-to-noise-ratios (SNRs) are summarized in Table 1 . It is observed from Table 1 that almost all 


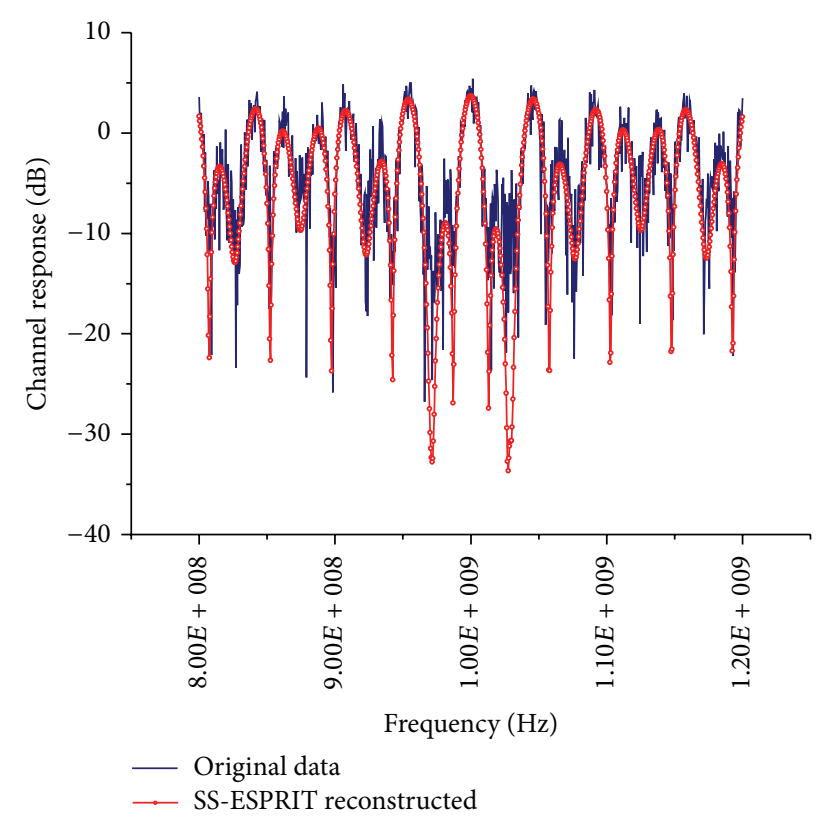

Figure 1: Comparison of the simulated channel response and the SS-ESPRIT reconstructed responses at $10 \mathrm{~dB}$ SNR.

these methods can perfectly estimate the channel parameters at high SNRs, but at low SNRs, the SS-ESPRIT approaches show a lower model order and a better antinoise performance. Figure 1 depicts a comparison example between the original channel frequency response and the SS-ESPRIT reconstructed responses, in which a good agreement can be found. Furthermore, another advantage of SS-ESPRIT is that almost no disturbance multipath components show up in the estimated channel impulse response at low SNRs.

\section{Measurement Campaign}

A chaotic yard of collapsed buildings and pieces of furniture, with an area of about $100 \mathrm{~m} \times 20 \mathrm{~m}$, was selected for measurements. The channel frequency responses were measured in two different scenarios along a designated path from $10 \mathrm{~m}$ to $28 \mathrm{~m}$ away from the transmitting (Tx) antenna at regular spatial intervals of $0.2 \mathrm{~m}$, as shown in Figure 2, in which the circle represents measurement point. At each distance, three locations at $0.5 \mathrm{~m}$ spacing were measured to gain enough spatial independent samples. Thus, a total of about 270 spatial samples at each scene were obtained. To simulate the mobile base station, the receiving $(\mathrm{Rx})$ antenna was mounted on a $4 \mathrm{~m}$ high pole, which was moved from one measurement point to another during the measurement. In the two scenarios, the Tx antenna was placed behind ruins and then covered by stratified media. To reduce the interference, all the mobile phones around were turned off, and an average of 200 snapshots for each sample was taken by vector network analyzer (VNA).

During the measurements, the channel responses from $700 \mathrm{MHz}$ to $2200 \mathrm{MHz}$ with an interval of $0.5 \mathrm{MHz}$ were recorded by R\&S ZVA67 VNA. Since the reciprocal of

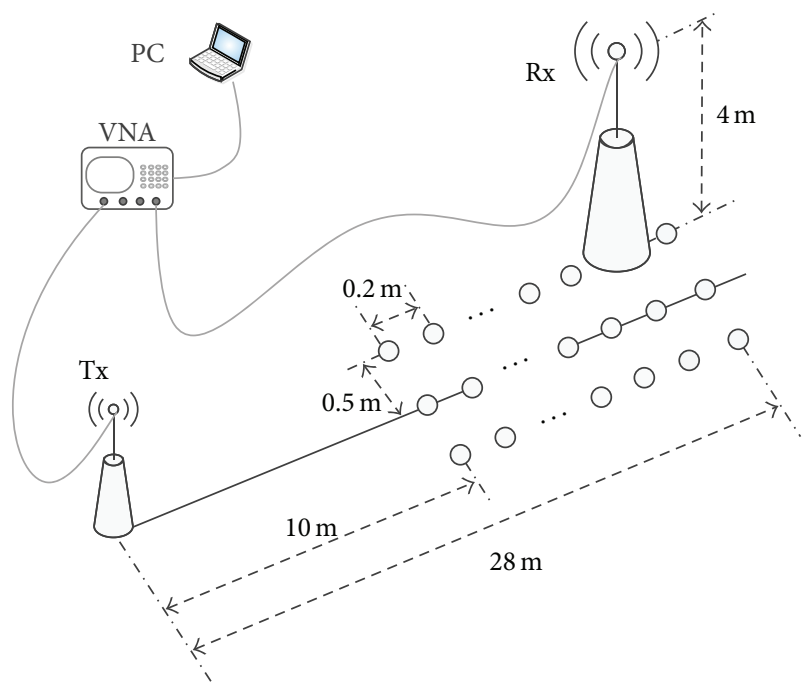

FIGURE 2: Diagram of the measurement layout.

frequency interval is the time length of signal, a $2 \mu$ s time window can be obtained for $0.5 \mathrm{MHz}$ interval, which is long enough to analyze the impulse responses in this study. Two zero gain omnidirectional biconical antennas, which were vertically polarized, were utilized to transmit and receive signal.

\section{Results and Discussion}

Radio channel in the test field is a static problem, which is in line with the scenarios after disaster. The time-varying factors, such as moving rescuers, are not taken into consideration in this study. As the disaster channel is complicated and time-invariant, it is viewed as a wide sense stationary uncorrelated scattering channel. Based on the experimental data, three mobile communication frequency bands are studied: 0.8-1 GHz, $1.7-1.9 \mathrm{GHz}$, and $1.9-2.1 \mathrm{GHz}$. A bandwidth of $200 \mathrm{MHz}$ is utilized for parameter estimation according to analysis of the measured data.

4.1. Performance of SS-ESPRIT Estimation. Figure 3 depicts a contrast example between the measured channel frequency response and the signal reconstructed by SS-ESPRIT method, in which almost all the measured peaks are covered, and an acceptable agreement can be found. Due to serious scattering and multipath interferences, a little error exists as well. The mean NRMSEs at different frequency bands are about 0.46 for SS-ESPRIT estimation, whereas they are about 0.55 for total least squares ESPRIT. As to other methods, the NRMSEs are much worse-around 1 or larger. In [6], the NRMSE varies from 0.3 to 0.45 , which is a little smaller than this work because of much less interference. For further evaluation of the SS-ESPRIT method, contrastive analysis between the Fourier and SS-ESPRIT estimated impulse responses is performed. A comparison of the estimated responses is given in Figure 4, and it indicates that impulse responses obtained by the two methods agree well with each other and a little 


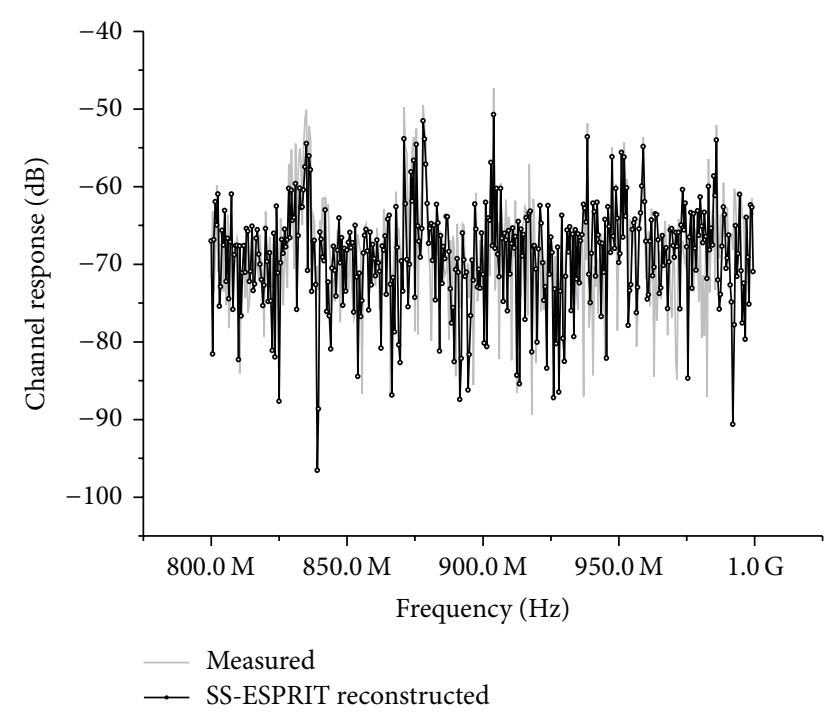

FIGURE 3: Comparison of the measured data and the SS-ESPRIT reconstructed signal in Scenario 1.

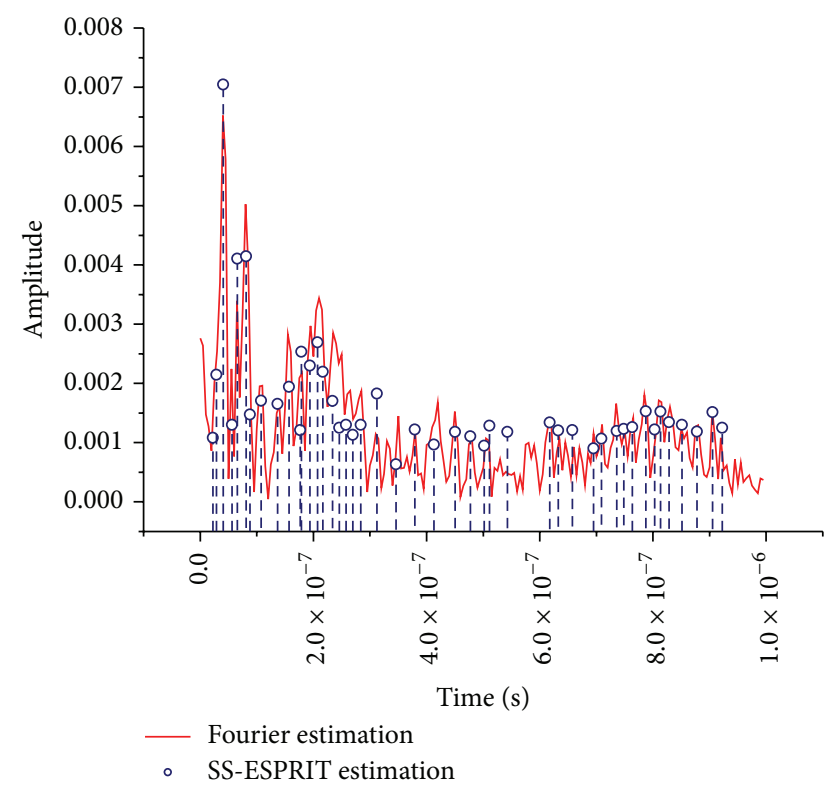

FIGURE 4: Comparison of Fourier and SS-ESPRIT estimated impulse responses at $900 \mathrm{MHz}, 11 \mathrm{~m}$, in Scenario 1.

deviation of the peaks mainly results from spectral leakage and interferences. Besides, it is found that a lot of small spurious interferences show up in the estimated channel impulse responses due to complex multipath fading, which leads to the bad performance of conventional modeling methods.

In order to reduce the unreasonable results, multipath components after $400 \mathrm{~ns}$, whose amplitudes are significantly small in all cases, are removed from the measured channel frequency responses by 15 th order Butterworth filter. Consequently, the SS-ESPRIT estimated impulse responses are truncated and the model orders are reduced from about 200

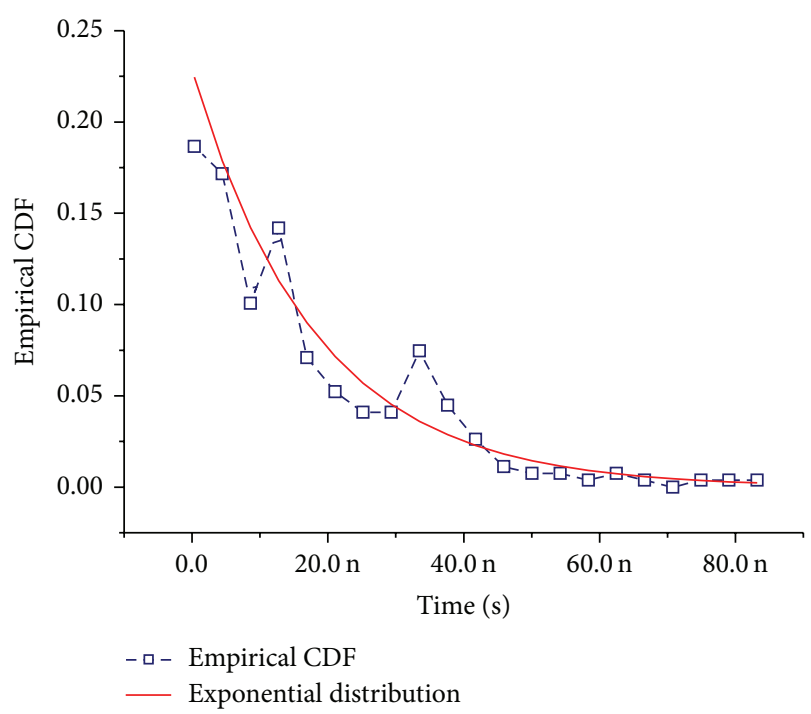

FIgURE 5: Empirical cumulative distribution and fitted exponential distribution of the excess delay of the first arrival at $2 \mathrm{GHz}$ in Scenario 1.

to 35 . To characterize the radio propagation behavior in ruins, statistical properties of the other three channel parameters, $a_{k}, \theta_{k}$, and $\tau_{k}$, are analyzed in the next part.

4.2. Statistical Results of the Delays and Amplitudes. In our investigation, the delays, amplitudes, and phases of multipath components, show no regularity, which are viewed as random variables, and statistical analysis is performed for characterization. Distribution functions (normal, exponential, Rayleigh, Nakagami, Rice, and Weibull) are applied to fit the aforementioned parameters, and the Akaike Information Criterion is utilized to compare the fitting performance. Besides, the best fitting distribution function for each parameter is validated by the Kolmogorov-Smirnov goodness-of-fit test. As the spatial samples are measured at different locations, the delays and amplitudes of multipath components should be normalized first. The normalized delays $\tau_{k}$, namely, excess delay, are obtained by subtracting the line-of-sight propagation delay from the measured $\tau_{k}$, whereas the amplitudes $a_{k}$ are normalized by dividing the theoretical values of free space propagation.

The excess delay of the first arrival $d_{\mathrm{fa}}$ is a very important channel parameter, especially for localization methods based on arrival time. As shown in Figure 5, statistical analysis indicates that exponential distribution fits the measured cumulative distribution function (CDF) of $d_{\mathrm{fa}}$ best; that is, $d_{\mathrm{fa}} \sim e(\lambda)$. Besides, the parameters of the fitted distributions are summarized in the third row in Table 2 . The mean value of exponential distribution is exactly the parameter $\lambda$ and it is found that the mean of $d_{\mathrm{fa}}$ is quite small. In general, if a lot of large obstacles show up, the arrival time would become longer. Furthermore, the normalized amplitudes of the first arrival $\bar{a}_{\mathrm{fa}}$ is normally distributed; that is, $\bar{a}_{\mathrm{fa}} \sim N(\mu, \sigma)$. And the parameters of the fitted normal distribution are summarized as well in the last two rows in Table 2. 
TABLE 2: Distribution parameters of the excess delay and the normalized amplitude of the first arrival.

\begin{tabular}{lccccc}
\hline Scene & \multicolumn{3}{c}{ Scenario 1} & \multicolumn{3}{c}{ Scenario 2} \\
Freq. $(\mathrm{GHz})$ & 0.9 & 1.8 & 2 & 0.9 & 1.8 \\
\hline$d_{\mathrm{fa}}-\lambda$ & $1.410 e-08$ & $2.173 e-08$ & $2.080 e-08$ & $7.781 e-09$ & $9.030 e-09$ \\
$\bar{a}_{\mathrm{fa}}-\mu$ & 0.0059 & 0.0086 & 0.0077 & 0.0164 & 0.0186 \\
$\bar{a}_{\mathrm{fa}}-\sigma$ & $1.647 e-03$ & $2.929 e-03$ & $2.442 e-03$ & $8.827 e-03$ & $7.635 e-03$ \\
\hline
\end{tabular}

TABLE 3: The normalized $a_{k}$, the parameters of the fitted Rayleigh distributions in two scenarios.

\begin{tabular}{ccccccc}
\hline Scenario & Freq. $(\mathrm{GHz})$ & $\begin{array}{c}\text { Group 1 } \\
<d_{\mathrm{fa}}\end{array}$ & $\begin{array}{c}\text { Group 2 } \\
-\left(d_{\mathrm{fa}}+20 \mathrm{~ns}\right)\end{array}$ & $\begin{array}{c}\text { Group 3 } \\
-\left(d_{\mathrm{fa}}+50 \mathrm{~ns}\right)\end{array}$ & $\begin{array}{c}\text { Group } 4 \\
-\left(d_{\mathrm{fa}}+250 \mathrm{~ns}\right)\end{array}$ & $\begin{array}{c}\text { Group 5 } \\
>d_{\mathrm{fa}}+250 \mathrm{~ns}\end{array}$ \\
\hline \multirow{3}{*}{1} & 0.9 & $2.262 e-03$ & $3.752 e-03$ & $3.285 e-03$ & $2.278 e-03$ & $1.913 e-03$ \\
& 1.8 & $3.677 e-03$ & $5.623 e-03$ & $4.962 e-03$ & $4.255 e-03$ & $3.108 e-03$ \\
& 2 & $3.509 e-03$ & $4.971 e-03$ & $4.243 e-03$ & $3.433 e-03$ & $2.589 e-03$ \\
\hline \multirow{2}{*}{2} & 1.9 & $4.990 e-03$ & $4.432 e-03$ & $2.237 e-03$ & $2.039 e-03$ & $1.510 e-03$ \\
& 1.8 & $3.888 e-03$ & $8.307 e-03$ & $4.833 e-03$ & $4.341 e-03$ & $2.989 e-03$ \\
& 2 & $3.531 e-03$ & $7.007 e-03$ & $3.850 e-03$ & $3.575 e-03$ & $2.588 e-03$ \\
\hline
\end{tabular}

In this investigation, no explicit relationship between the normalized amplitudes and $d_{\mathrm{fa}}$ is found and the expected phenomenon in which the amplitudes decrease with increasing $d_{\mathrm{fa}}$ does not show up. Moreover, the signal energy is larger around the arrival time and it tends to reduce after arrival. Therefore, to describe the behavior of the normalized $a_{k}$ elaborately, the $400 \mathrm{~ns}$ time window is divided into five groups according to the difference between the excess delay and $d_{\mathrm{fa}}$. Among these groups, given in Table 3, Group 2 is the arrival time interval. Besides, the time length of Group 4 is set very large as subdivided groups in this range obey almost the same distribution. As shown in Figure 6, the best description of the normalized $a_{k}$ in each group is Rayleigh distribution and Table 3 summarizes the corresponding distribution parameters under different conditions. Because of more serious blocking effect, the amplitudes of Group 2 in Scenario 1 are much smaller than in Scenario 2.

In addition, the phases of multipath components $-\theta_{k}$ in each group are uniformly distributed in the range $[0,2 \pi]$ in this study. Besides, the excess delay of each multipath component obeys uniform distribution as well in the observed time interval.

4.3. Building and Validation of the Channel Simulator. Simulation of radio channel is always a research hotspot, which can help configure the localization system and evaluate the performance of base station to mobile station communication as well as localization. Based on the statistical analysis of the parameters of channel impulse responses, a channel simulator is built and here are the steps.

(1) First, the normalized $a_{k}$ and excess delay of the first arrival are determined as illustrated in Table 2.

(2) Then, a set of normalized $a_{k}$ in each interval group is generated, according to the Rayleigh parameters in Table 3.

(3) Afterwards, a total of $K$ excess delays, which are uniformly distributed from 0 ns to about 400 ns, are

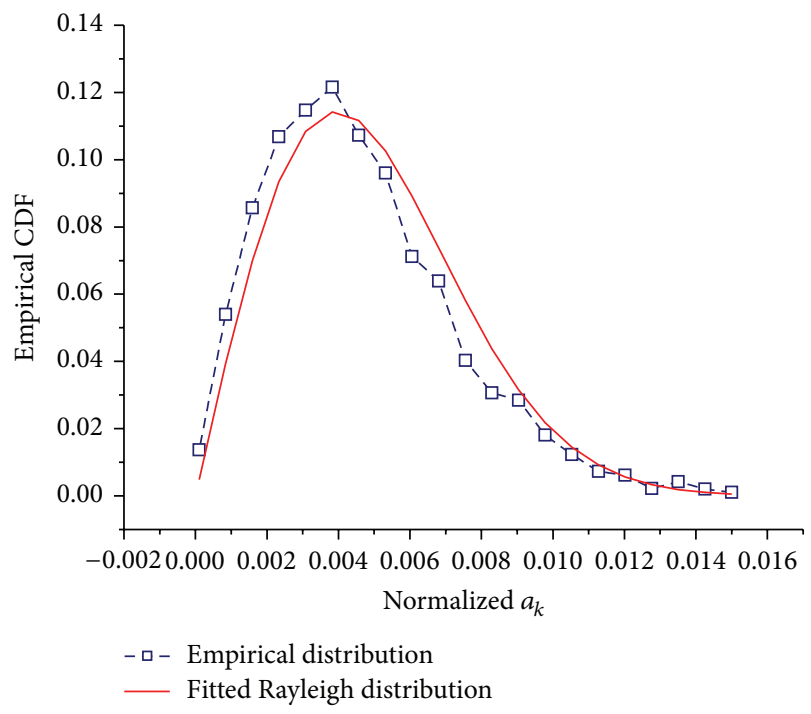

FIGURE 6: Empirical cumulative distribution and fitted Rayleigh distribution of the $a_{k}$ in Group 4 at $1.8 \mathrm{GHz}$ in Scenario 2.

given. $K$ is the number of multipath components, namely, 35 in this study. Besides, the phases $\theta_{k}$, which are evenly distributed in the range $[0,2 \pi]$, are generated in the same way.

(4) The excess delays obtained in Step 3 are classified according to the aforementioned groups. In each group, the corresponding normalized amplitudes $a_{k}$ are determined by choosing values of the same amount in the data set generated in Step 2.

(5) Finally, the "normalized" channel impulse response is constructed based on the multipath channel model in (1).

To characterize the radio propagation in ruins and validate the channel model, two important channel parameters 
TABLE 4: Average excess delay and delay spread, the parameters of the fitted normal distributions.

\begin{tabular}{|c|c|c|c|c|c|c|}
\hline \multirow{2}{*}{ Scenario } & \multirow{2}{*}{ Freq. (GHz) } & \multirow{2}{*}{ Parm. } & \multicolumn{2}{|c|}{ Average excess delay } & \multicolumn{2}{|c|}{ Delay spread } \\
\hline & & & Meas. & Regenerate & Meas. & Regenerate \\
\hline \multirow{6}{*}{1} & \multirow{2}{*}{0.9} & $\mu$ & $1.250 e-07$ & $1.247 e-07$ & $1.031 e-07$ & $1.015 e-07$ \\
\hline & & $\sigma$ & $2.501 e-08$ & $2.345 e-08$ & $8.412 e-09$ & $9.063 e-09$ \\
\hline & \multirow{2}{*}{1.8} & $\mu$ & $1.375 e-07$ & $1.372 e-07$ & $1.109 e-07$ & $1.021 e-07$ \\
\hline & & $\sigma$ & $2.129 e-08$ & $2.195 e-08$ & $8.976 e-09$ & $9.709 e-09$ \\
\hline & \multirow{2}{*}{2} & $\mu$ & $1.336 e-07$ & $1.304 e-07$ & $1.021 e-07$ & $9.938 e-08$ \\
\hline & & $\sigma$ & $2.119 e-08$ & $2.135 e-08$ & $9.001 e-09$ & $9.637 e-09$ \\
\hline \multirow{6}{*}{2} & \multirow{2}{*}{0.9} & $\mu$ & $6.901 e-08$ & $6.663 e-08$ & $9.021 e-08$ & $8.157 e-08$ \\
\hline & & $\sigma$ & $3.013 e-08$ & $2.580 e-08$ & $2.331 e-08$ & $1.589 e-08$ \\
\hline & \multirow{2}{*}{1.8} & $\mu$ & $1.018 e-07$ & $1.012 e-07$ & $1.007 e-07$ & $9.590 e-08$ \\
\hline & & $\sigma$ & $2.709 e-08$ & $2.305 e-08$ & $9.291 e-09$ & $1.074 e-08$ \\
\hline & \multirow{2}{*}{2} & $\mu$ & $1.209 e-07$ & $1.178 e-07$ & $1.043 e-07$ & $1.014 e-07$ \\
\hline & & $\sigma$ & $2.235 e-08$ & $2.327 e-08$ & $9.397 e-09$ & $1.073 e-08$ \\
\hline
\end{tabular}

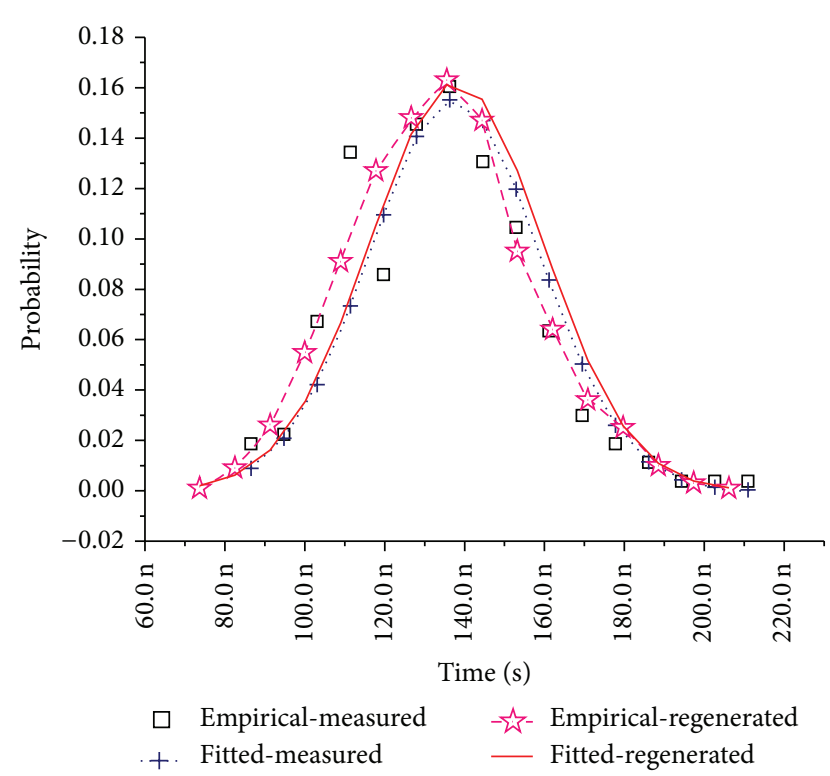

Figure 7: Comparison of the empirical distribution and fitted normal distribution of excess delay at $1.8 \mathrm{GHz}$ in Scenario 1. "Measured" means that the data were obtained by experiments whereas "regenerated" means that they were obtained by channel simulator.

(average excess delay and delay spread) are analyzed. Two examples of statistical analysis results are illustrated in Figures 7 and 8, which show that the best descriptions of the average excess delay and delay spread are normal distribution $(N(\mu, \sigma))$ for both the measured and regenerated channel impulse responses, and all the statistical parameters are summarized in Table 4. For average excess delay, the measured parameters show a good agreement with the regenerated parameters. From Table 4, it is found that the average excess delay in Scenario 1 is about $130 \mathrm{~ns}$, which is slightly higher than Scenario 2 due to more serious blocking effect. As to delay spread, a little deviation of the regenerated data (about several nanoseconds) is found in Table 4 , which

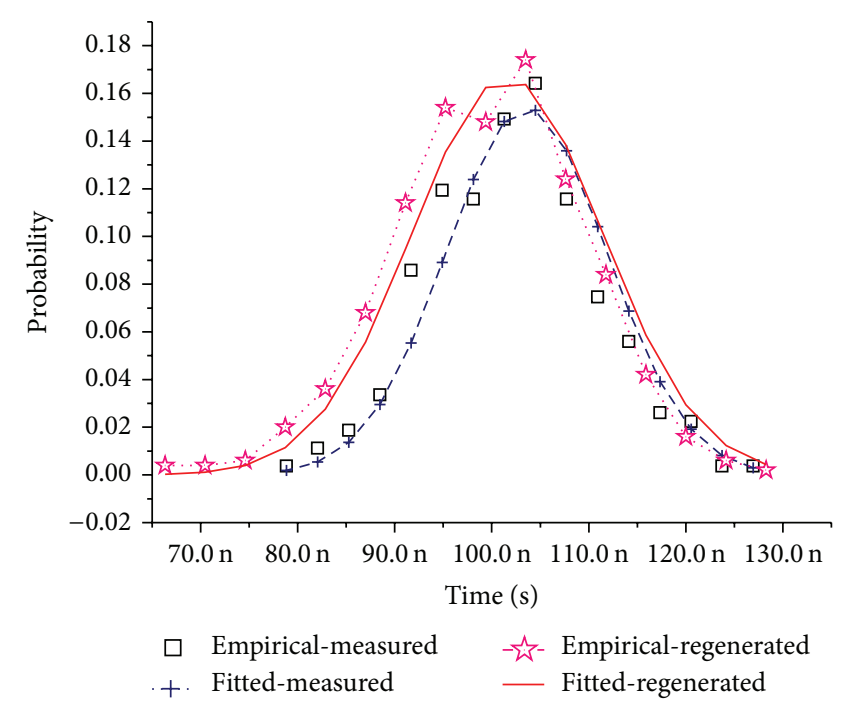

FIgURE 8: Comparison of the empirical distribution and fitted normal distribution of delay spread at $900 \mathrm{MHz}$ in Scenario 1. "Measured" means that the data were obtained by experiments whereas "regenerated" means that they were obtained by channel simulator.

is within about $10 \%$. Generally, the error is acceptable for simulation analysis of channel characteristics. In addition, the delay spread values in the two scenarios are both about $100 \mathrm{~ns}$.

\section{Conclusion}

In this paper, a SS-ESPRIT based characterization of radio propagation channel in high density ruins is presented. By measurements in a large yard full of collapsed buildings, the radio propagation channel has been investigated at three mobile communication bands $(0.9,1.8$, and $2 \mathrm{GHz})$ and the results show the SS-ESPRIT method is applicable for such analysis. Furthermore, the delays, amplitudes, and phases of multipath components are statistically analyzed and different distribution functions are applied for description of these 
parameters. Based on these results, a channel simulator is established and it is validated through the analysis of two key channel parameters (average excess delay and delay spread) where the experimental data show an acceptable agreement with the channel modeling results. As a conclusion, this paper provides an effective method for modeling of communication channel in high density ruins, and the analysis results reported in the paper can be utilized for design of the phonebased localization system.

\section{Conflict of Interests}

The authors declare that there is no conflict of interests regarding the publication of this paper.

\section{References}

[1] S. Zorn, G. Bozsik, R. Rose, A. Goetz, and R. Weigel, "A novel technique for mobile phone localization for search and rescue applications," in Proceedings of the International Conference on Indoor Positioning and Indoor Navigation (IPIN '10), pp. 1-4, Zürich, Switzerland, September 2010.

[2] J. He, L. S. Chai, X. C. Wei, and E. P. Li, "Radio frequency channel characterization of a novel localization system for rescue in disaster scenarios," in Proceedings of the General Assembly and Scientific Symposium of the International Union of Radio Science, Beijing, China, 2014.

[3] C. Chen, Y. Guo, Q. Xie, and Z. Xiao, "A new life detecting system research and design based on cell phone detecting," Instrumentation Customer, vol. 15, no. 3, pp. 12-13, 2008.

[4] L. S. Chai, Research of radio channel based on a localization rescue system in disasters [M.S. thesis], Department of Information Sciences and Electrical Engineering, Zhejiang University, Hangzhou, China, 2014.

[5] A. DiCarlofelice, E. DiGiampaolo, M. Feliziani, and P. Tognolatti, "Experimental characterization of electromagnetic propagation under rubble of a historic town after disaster," IEEE Transactions on Vehicular Technology, 2014.

[6] L. Chen, M. Loschonsky, and L. M. Reindl, "Autoregressive modeling of mobile radio propagation channel in building ruins," IEEE Transactions on Microwave Theory and Techniques, vol. 60, no. 5, pp. 1478-1489, 2012.

[7] S. J. Howard and K. Pahlavan, "Autoregressive modeling of wide-band indoor radio propagation," IEEE Transactions on Communications, vol. 40, no. 9, pp. 1540-1552, 1992.

[8] S. Haykin, Adaptive Filter Theory, Publishing House of Electronics Industry, Beijing, China, 4th edition, 2006.

[9] G. Morrison and M. Fattouche, "Super-resolution modeling of the indoor radio propagation channel," IEEE Transactions on Vehicular Technology, vol. 47, no. 2, pp. 649-657, 1998.

[10] D. W. Tufts and R. Kumaresan, "Estimation of frequencies of multiple sinusoids: making linear prediction perform like maximum likelihood," Proceedings of the IEEE, vol. 70, no. 9, pp. 975-989, 1982.

[11] L. Qu, Q. Sun, T. Yang, L. Zhang, and Y. Sun, "Time-delay estimation for ground penetrating radar using ESPRIT with improved spatial smoothing technique," IEEE Geoscience and Remote Sensing Letters, vol. 11, no. 8, pp. 1315-1319, 2014.

[12] C. L. Bastard, V. Baltazart, Y. Wang, and J. Saillard, "Thinpavement thickness estimation using GPR with high-resolution and superresolution methods," IEEE Transactions on Geoscience and Remote Sensing, vol. 45, no. 8, pp. 2511-2519, 2007.

[13] B. Ottersten, M. Viberg, and T. Kailath, "Performance analysis of the total least squares ESPRIT algorithm," IEEE Transactions on Signal Processing, vol. 39, no. 5, pp. 1122-1135, 1991.

[14] A. L. Wang, ESPRIT parameter estimation algorithm in wideband channel system [M.S. thesis], Nanjing University of Posts and Telecommunications, Nanjing, China, 2012.

[15] J. Rissanen, "Modeling by shortest data description," Automatica, vol. 14 , no. 5, pp. 465-471, 1978. 

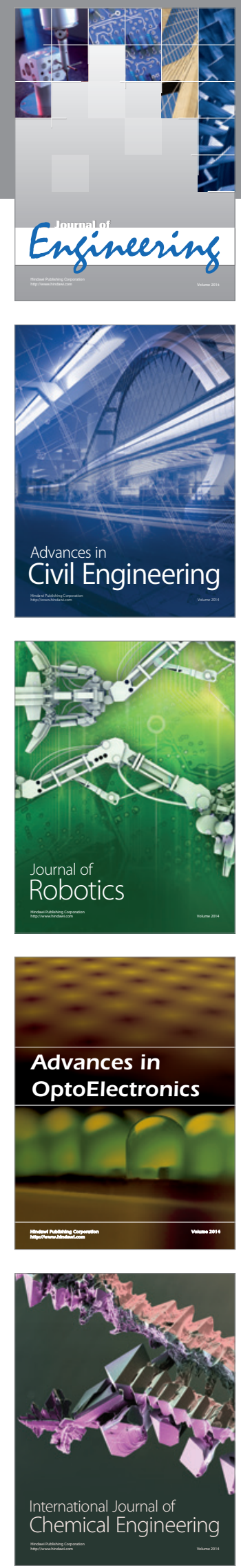

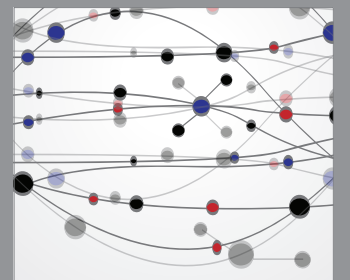

The Scientific World Journal
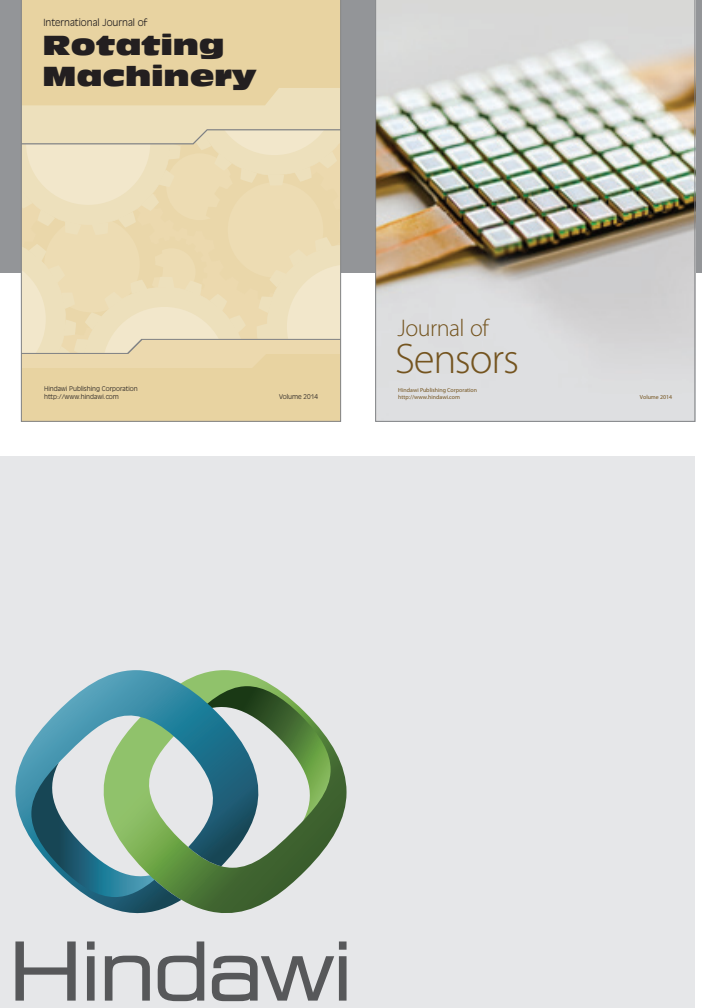

Submit your manuscripts at http://www.hindawi.com
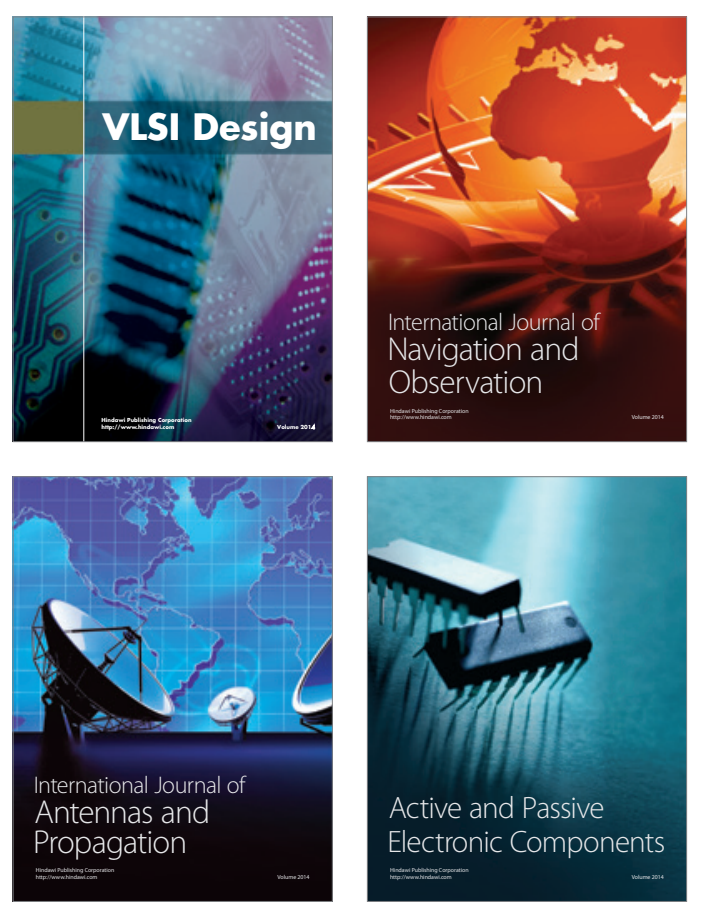
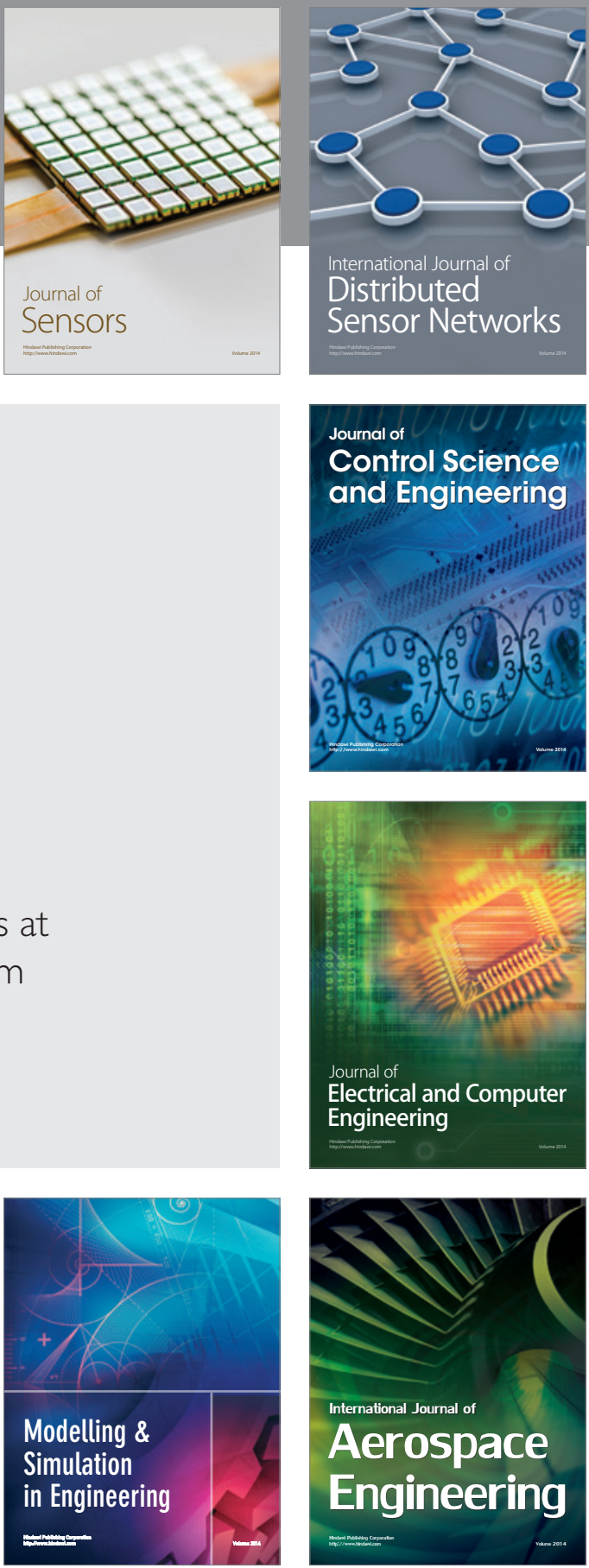

Journal of

Control Science

and Engineering
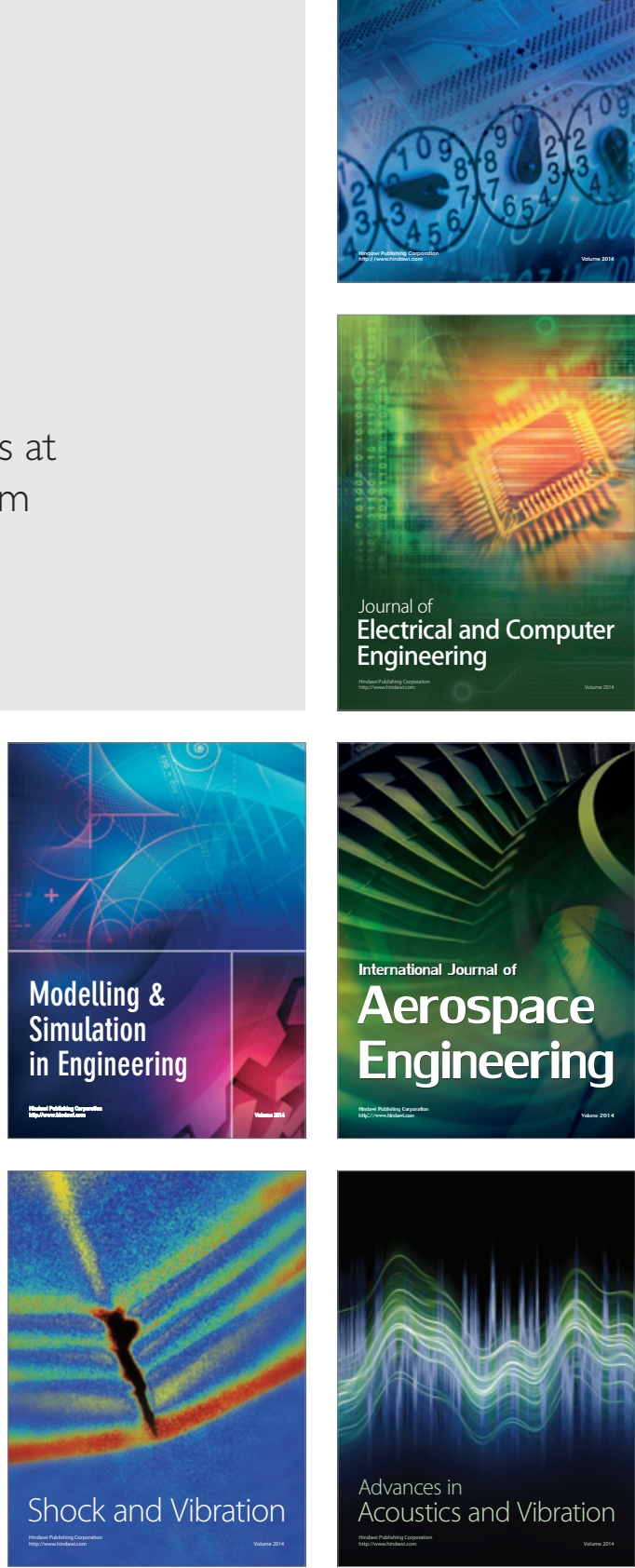